\title{
Retroperitoneal Sarcoma - A Clinico-Pathological Study
}

\author{
Siva Ranjith, Sulfekar MS, Subhash \\ Department Of General Surgery, Govt. Medical College Trivandrum \\ Correspondences: Dr.Siva Ranjith, Senior Resident, Division of Surgical Oncology, Regional Cancer Centre, \\ Trivandrum, Kerala. PIN: 695011
}

\begin{abstract}
Retroperitoneal sarcomas are rare tumours that comprise approximately $15 \%$ of all soft tissue sarcomas in adults ${ }^{l}$. Most of the retroperitoneal tumours are malignant and approximately one third is soft tissue sarcoma. Although significant advances in our understanding of extremity soft tissue sarcoma have resulted in improved treatments and outcomes, similar progress has not been achieved in our understanding and treatment of retroperitoneal soft tissue sarcomas. Here we present a retrospective analysis of 30 cases of retroperitoneal sarcoma from a tertiary care centre from 2006 to 2010. The incidence of retroperitoneal sarcoma in our study was around $0.10 \%$ of the total surgical admissions. Peak incidence was seen between 5060 years and $>85 \%$ patients were above 40 years. There was a definite male preponderance, with a male to female ratio of 2.75: 1. The most useful and economical investigative modality is contrast enhanced CT scan of the abdomen Most of the patients present at a later stage of the disease with adjacent organ involvement and distant metastasis. These factors contribute to the bad prognostic nature of the disease. Potentially curative resection in the form of en-bloc resection/ complete excision was possible only in 50\% of cases. The role of adjuvant treatment in retroperitoneal sarcoma still remains unsettled. The most common histopathological type of retroperitoneal sarcoma was liposarcoma. Majority of patients developed local recurrence and distant metastasis within a short period, especially in margin compromising resections. Margin negative resections are associated with good prognosis.
\end{abstract}

Keywords: Retro-peritoneal Sarcoma, Clinico-pathological

\section{Introduction}

Retroperitoneal sarcomas are rare tumours that comprise approximately $15 \%$ of all soft tissue sarcomas in adults [1]. Most of the retroperitoneal tumours are malignant and approximately one third is soft tissue sarcoma. The peak incidence of most tumours is during the fifth and sixth decades of life. The most common sarcomas occurring in the retroperitoneum are liposarcoma, malignant fibrous histiocytoma and leiomyosarcomas.

The diagnosis is usually established by CT scan of the abdomen and pelvis. The histological diagnosis is not required before surgery, other than to rule out lymphoma or germ cell neoplasm, or when neo-adjuvant therapy is contemplated. Complete surgical excision remains the primary curative treatment for these tumours. The role of adjuvant treatment in the management remains controversial.

Although significant advances in our understanding of extremity soft tissue sarcoma have resulted in improved treatments and outcomes, similar progress has not been achieved in our understanding and treatment of retroperitoneal soft tissue sarcomas [2]. Due to several reasons, patients with retroperitoneal soft tissue sarcomas generally have a worse prognosis than those with extremity sarcomas. One major reason is that retroperitoneal soft tissue sarcomas commonly grow to large sizes before they become clinically apparent, by which time they often involve vital structures, which precludes surgical resection. A second reason is that the surgical margins that can be obtained around these sarcomas are often inadequate because of anatomic constraints.

In an analysis [3] of 500 patients with retroperitoneal soft tissue sarcoma treated at Memorial SloanKettering cancer centre, the 5 year over all survival rate was 54\%. The median survival was 103 months for those who underwent complete resection versus 18 months for patients who underwent incomplete resection.

\section{Materials And Methods}

Primary aim of our study was to find the proportion and clinical presentation of retroperitoneal sarcoma in a tertiary care centre. The secondary aims were to find the distribution of various histopathological types and grade of retroperitoneal sarcoma and to evaluate the diagnosis, management, post operative complications and prognosis of retroperitoneal sarcoma.

This is a retrospective case series from Department of General Surgery, Medical College, Thiruvananthapuram. All patients, above 12 years, with a provisional diagnosis of retroperitoneal tumour based on clinical and radiological features registered in the institution from January 2006 to January 2010, for a period 
of four years, were considered for the study. Thirty-nine Patients were thus included initially. All retroperitoneal tumours with tissue diagnosis other than sarcoma, proved later in the course of treatment, were excluded from the study and thus nine patients were excluded from the study. So a total of 30 histologically proven cases of retroperitoneal sarcoma were finally eligible for the study. Data was analyzed using computer software, Statistical Package for Social Sciences (SPSS) version 10.

\section{Observations And Results}

The proportion of retroperitoneal sarcoma in our series was $0.097 \%$ (approximately $0.1 \%$ ) of the total surgical admissions. Peak incidence in our series was between 50 - 60 years, with a male to female ratio of 2.75: 1. The most common presenting symptom was fullness or lump abdomen which was seen in $17(56.7 \%)$ patients. The most consistent clinical sign was a lump abdomen, in 24(80\%) patients. The most useful and economical investigative modality was contrast enhanced CT scan (CECT) of the abdomen with an efficacy of 93.2\%. The efficacy of MRI was $100 \%$, eventhough it was taken in only 2 patients. However, considering the cost and availability, CT remains the diagnostic procedure of choice. Only 10 (33.3\%) cases had disease cofined to the site of origin (Figure 1). Potentially curative resection in any form, i.e. either an enbloc resection or a complete resection was possible only in $15(50 \%)$ cases (Figure 2).

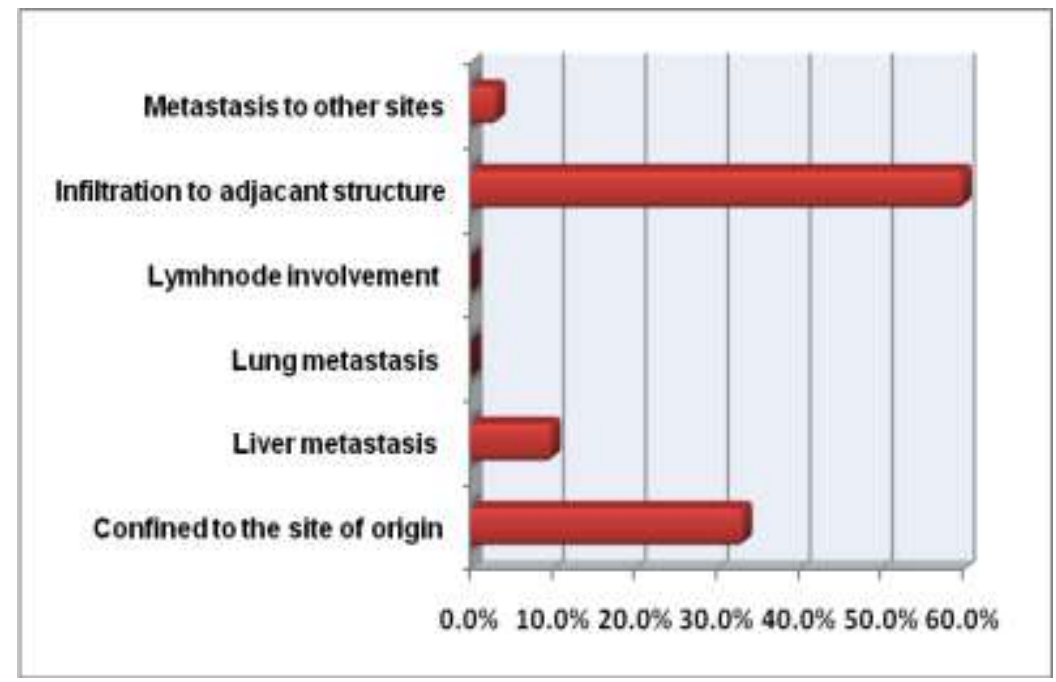

Figure 1: Extent of disease

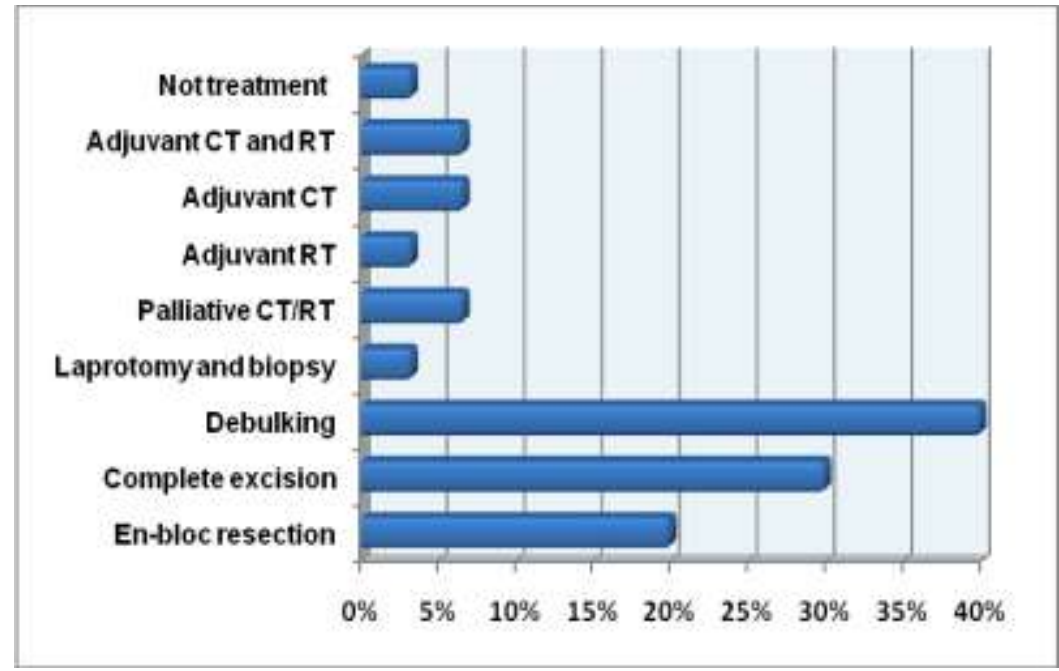

Figure 2: Treatment offered

Gross specimens when pathological analysed, the size was more than 5 centimetres in all the cases. Most common histology was liposarcoma in 46.6\% (Table 1). In 20 (66.7\%) cases the lesions were high grade when microscopically analysed and that added to the bad prognosis of the disease. When the resected specimens were microscopically studied, only $5(18.5 \%)$ had tumour free margins. In rest of the 25 cases, the margins were positive, either grossly or microscopically. 
Table 1: Distribution of Histopathological types

\begin{tabular}{|l|l|l|}
\hline HPR & Number & Percentage \\
\hline Liposarcoma & 14 & $46.66 \%$ \\
\hline Leiomyosarcoma & 3 & $10 \%$ \\
\hline Fibrosarcoma & 1 & $3.33 \%$ \\
\hline MPNST & 5 & $16.66 \%$ \\
\hline MFH & 5 & $16.66 \%$ \\
\hline PNET & 1 & $3.33 \%$ \\
\hline $\begin{array}{l}\text { Mesenchymal } \\
\text { chondrosarcoma }\end{array}$ & 1 & $3.33 \%$ \\
\hline \multicolumn{2}{|l|}{$\begin{array}{l}\text { MPNST - Malignant Peripheral Nerve Sheath Tumour; MFH - Malignant } \\
\text { Fibrous Histiocytoma; PNET - Peripheral Neuro Ectodermal Tumours }\end{array}$} \\
\hline
\end{tabular}

In $19(63.3 \%)$ cases some form of complications were there during admission period. Of which, majority were due to co-morbidities that complicated the postopertive period. Only one in-hospital mortality. On follow up, 10 (33.3\%) patients developed local recurrence and 7 (23.3\%) had distant metastasis. Five (16.7\%) patients were dead during this period and $3(10 \%)$ were lost to follow up. Only $5(16.7 \%)$ patients were disease free.

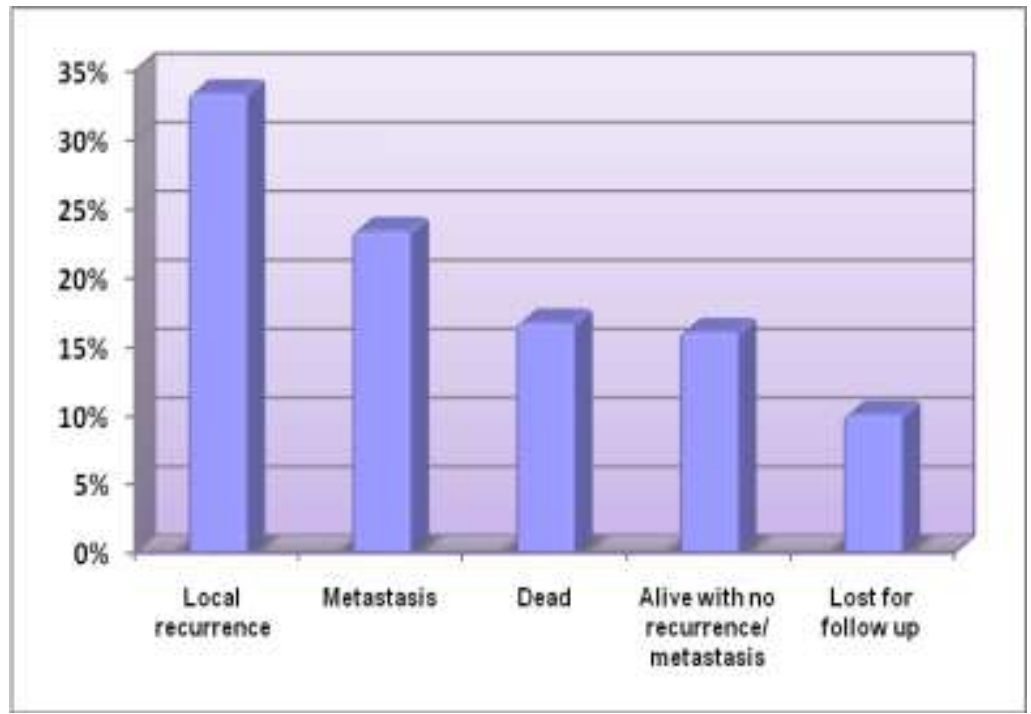

Figure 3: Follow Up

\section{Discussion}

In this series of retroperitoneal sarcoma, initially a total of 39 patients were included, but later 9 patients were excluded based on exclusion criteria. The analysis of recorded observations regarding the final 30 cases of retroperitoneal sarcoma has shown that the result generally tallies with similar studies in the literature. There are certain limitations in our study. The study has not included paediatric cases and hence the comparison of overall incidence, incidence in different age groups and distribution of different histopathological types of the disease with other studies may not be that accurate. Further the follow up period are too short to draw any tangible conclusions from the results, with regard to prognosis. The overall incidence of retroperitoneal sarcoma in our hospital during the 4 year period analyzed shows a range from $0.095 \%$ to $0.099 \%$ of all the surgical admissions and that agrees with the incidence in literature. With regard to the age incidence, peak incidence in this series is found to be between 50 - 60 years. This is in consensus with the following reports.

\begin{tabular}{|l|l|l|}
\hline \multicolumn{1}{|c|}{ Study } & Number Of Cases & Median Age (Years) \\
\hline Lewis et al., 1998 [3] & 500 & 58 \\
\hline Stocke1e et al., 2001 [4] & 165 & 54 \\
\hline
\end{tabular}

In this series it is clear that the most common presenting symptom of the patient was lump abdomen or abdominal fullness $(56.67 \%)$ and the second common feature being pain abdomen $(30.00 \%)$. This may be due to the fact that the tumors remain asymptomatic until they grow large enough to compress or invade contiguous structures to produce any symptoms.

The most useful tool in evaluation was CT scan; which allows assessment of tumor location, local extension and metastasis. USG and CT scan were done in all cases and they were $60 \%$ and $93.2 \%$ accurate in 
diagnosis and anatomical delineation respectively. But they often fail to give an accurate tissue diagnosis which is possible only with image guided biopsy or laparotomy and biopsy. MRI was needed in two cases suspecting neurovascular invasion and image guided biopsy was offered in two cases, first to rule out lymphoma and another case which was inoperable.

The extent of the disease at the time of diagnosis was studied and it was found that in majority of cases tumor has extended to involve adjacent structures $(60 \%)$ and in $13.3 \%$ cases some form of metastasis was there. Only $33.3 \%$ presented at a comparatively early stage with no metastasis or adjacent organ involvement. These results are comparable to the studies of Alvarengo et al., 1991[5] and Priyesh et al., 2001[6].

Laparotomy was under taken in majority of cases $(93.2 \%)$ of retroperitoneal sarcoma but en-bloc resection or complete excision was possible only in 50\% cases (Figure 2). In majority of cases (40\%) partial excision was done; mainly to relieve pressure symptoms. Laparotomy and biopsy was done in one case. Of the $10 \%$ inoperable cases, two patients were sent for palliative treatment after histological confirmation. These observations made in our study are similar to the following series in medical literature.

\begin{tabular}{|l|l|l|}
\hline Study & Number Of Cases & Complete Resection (\%) \\
\hline Priyesh et aI.,2001[6] & 22 & 41 \\
\hline Dalton et al., 1990 [7] & 116 & 54 \\
\hline Catton et al., 1994 [8] & 104 & 43 \\
\hline
\end{tabular}

All the patients were subsequently referred to Radiotherapy department where only a few (16.67\%) undergone any form of adjuvant treatment. The analysis of adjuvant treatment plans in different patients showed absence of a fixed systematic protocol.

The histopathological analysis of specimens revealed liposarcoma in majority of cases (46.7\%) in our series, which tallies with most of other studies in literature. A huge study of 1111 patients by Porter G A et al., 2003 [9] proves the same. But a significant finding in our series is the increased incidence of MPNST, which was found in $16.7 \%$ cases. A comparatively low incidence of MFH (16.66\%) may be due to the routine use of immunohistochemical studies to know the exact tissue diagnosis in affordable patients.

Macroscopically $100 \%$ of the lesions were $>5 \mathrm{~cm}$ in size which is comparable to several other studies. The majority of lesions were also belonging to high grade $(66.8 \%)$ as in the following studies.

\begin{tabular}{|l|l|l|}
\hline Study & Size $>\mathbf{5 ~ C m ~ ( \% ) ~}$ & Grade (\%) \\
\hline Lewis et al., 1998 [3] & 94.00 & 60.00 \\
\hline Dalton et al., 1990 [7] & 98.00 & 54.00 \\
\hline Jaques et al., 1990 [10] & 100.00 & 57.00 \\
\hline Hassan et al.,2004 [12] & 97.00 & 69.00 \\
\hline
\end{tabular}

Resection margins were when analyzed microscopically, negative margins were observed only in $18.5 \%$ cases. The study by Lewis et al [3] has conclusively proved the importance of negative margin in the prognosis of the disease; which in case of a retroperitoneal sarcoma is impossible to ascertain in most of the cases as in our series.

Regarding the follow up of the cases there were certain limitations in our series that the follow up period was too short to draw any reasonable conclusions or to compare with other series in literature and few $(10 \%)$ patients were lost for follow up. Any way the analysis of data collected revealed a bad prognosis with $16.7 \%$ of patient dead already and $23.3 \%$ with metastasis and $33.3 \%$ with local recurrence. Only $16.7 \%$ are alive with no recurrence or metastasis.

\section{Conclusions}

The incidence of retroperitoneal sarcoma our area is around $0.10 \%$ of the total surgical admissions. Peak incidence is seen between 50-60 years and $>85 \%$ patients are above 40 years. There is definite male preponderance. The most common presenting symptom in patients is fullness/lump abdomen and the most consistent clinical sign is lump abdomen. The most useful and economical investigative modality is contrast enhanced CT scan of the abdomen. MRI is needed only in selected cases of doubtful neurovascular involvement. Even though the efficacy of MRI is high considering the cost and availability ct remains the diagnostic procedure of choice. Also biopsy is not mandatory before surgical treatment. Most of the patients present at a later stage of the disease with adjacent organ involvement and distant metastasis. This factor contributes to the bad prognostic nature of the disease. Potentially curative resection in the form of en-bloc resection/ complete excision is possible only in $50 \%$ of cases. In majority of cases the treatment offered are palliative resections. The role of adjuvant treatment in retroperitoneal sarcoma still remains unsettled. The most common histopathological type of retroperitoneal sarcoma is liposarcoma. In most of the cases tumour is $>5 \mathrm{~cm}$ and high grade, that adds to the bad prognosis of the disease. Margin negative resections are possible only in limited number of cases. Majority of patients develop local recurrence and distant metastasis within a short 
period, especially in margin compromising resections. Margin negative resections are associated with better prognosis.

\section{References}

[1]. Barry W. Feig. Retroperitoneal sarcoma. Surg oncol din N Am 12 (2003) p.369-377.

[2]. Barry W Feig, David H Berger, editors. The MD Anderson surgical oncology Hand book, 4th edition. Lippincott. 2006. p. 121-142.

[3]. Lewis JJ, Leung D, Woodruff JM, Brennan ME. Retroperitoneal sarcoma: analysis of 500 patients treated and followed at a single institution. Ann Surg 1998; 228:355-65.

[4]. Stockelle et al. prognostic factors in retroperitoneal sarcomas: a multivariate analysis of a series of 165 patients of FCCFSG. Cancer 2001; 92(2):359-368.

[5]. Alvarenga JC, Ball ABS, Fisher C et al. Limitations of surgery in the treatment of retroperitoneal sarcoma. Br J Surg 1991; 78:9126.

[6]. Priyesh A et al. The management of retroperitoneal sarcomas: a single institution experience. Eur J Surg Onco12001; 27:491-497.

[7]. Dalton RR, Donohue JH, Mucha P Jr; et a1. Management of retroperitoneal sarcomas. Surgery 1989;106:725-33.

[8]. Catton CN et al. Out come and prognosis in retroperitoneal sarcomas. Int J Radiat Onco1 BioI Phys. 1994; 29(5):1005-1010.

[9]. Porter GA et al. Retroperitoneal sarcoma. In: Saclarides TJ, editor. Surgical oncology: an algorithmic approach. New York: Springer 2003.p.390-5.

[10]. Jacques d et al, management of primary and recurrent retroperitoneal sarcoma. Ann Surg 1990; 212:51-59.

[11]. Hassan et al. Operative management of retroperitoneal sarcomas: a reappraisal. Ann Surg 2004; 239(2):244-250.

[12]. Richard H turnage et al., the abdominal wall, peritoneum and retroperitoneum. Editors. Sabiston Textbook of Surgery, $17^{\text {th }}$ edn, Elsevier 2004. P. 1194-97.

[13]. Pisters et al. Soft tissue sarcoma in Surgery basic science and clinical evidence, Newyork, springer 2001 norton JA, Bollinger et al.eds.

[14]. Brady MS et al. Radiation associated sarcoma of bone and soft tissue Arch Surg 1992; 127:1379-1385.

[15]. Helman LT, Meltzer P.Mechanisms of sarcoma development. Nay Rev 2003; 3:685-694.

[16]. Boring CC et a1.Cancer statistics, 1994, CA Cancer J Clin 1994; 44:7-26.

[17]. Cody HS et a1.The continuing challenge of RPS Cancer 1981; 47:2147-54. 